\title{
РАДИОЭЛЕКТРОННЫЕ СИСТЕМЫ
}

\section{УДК 621.396.96: 551.501}

В.К. ВОЛОСЮК, д-р техн. наук, С.С. ЖИЛА, канд. техн. наук, А.Д. СОБКОЛОВ, В.В. ПАВЛИКОВ, д-р техн. наук, Н.В. РУЖЕНЦЕВ, д-р техн. наук, Д.С. САЛЬНИКОВ, А.А. МЕРЗЛИКИН, А.И. ЦОПА, д-р техн. наук

\section{АППАРАТУРНО-МЕТОДИЧЕСКОЕ ОБЕСПЕЧЕНИЕ НАУЧНО-ПРИКЛАДНОЙ РАДИОМЕТРИИ АТМОСФЕРЫ}

\section{Введение}

Аппаратурно-методическое обеспечение метода радиометрии позволяет получать данные о радиояркостной температуре $\mathrm{T}_{\mathrm{b}}$ атмосферы и затем восстанавливать значения оптической толщины атмосферы $\tau$ и полного вертикального поглощения радиоволн.

Обе величины $\mathrm{T}_{\mathrm{b}}$ и $\tau$ связаны известным выражением для нисходящего радиотеплового излучения атмосферы:

$$
\mathrm{T}_{\mathrm{b}}=\mathrm{T}_{\text {eff }}\left(1-\mathrm{e}^{\tau \sec \theta}\right),
$$

где $\mathrm{T}_{\text {eff }}$ - это эффективная температура атмосферы, $\theta$ - зенитный угол визирования антенны радиометра.

Знание величины полного вертикального поглощения радиоволн на разных частотах позволяет определять разнообразные физические параметры атмосферы и некоторых ее составляющих [1]. Восстановление физических параметров атмосферы по данным дистанционного зондирования (ДЗ) методами радиометрии в миллиметровом диапазоне волн (ММ ДВ) имеет практический и научный интерес для метеорологии, климатологии, исследований физики облаков, искусственного подавления и стимуляции осадков. Эти методы хорошо развиты и нашли широкое практическое применение в мире за последние несколько десятилетий.

В последние годы появились новые приоритетные направления и задачи прикладного характера, новые возможности в развитии аппаратурного обеспечения радиометрических наблюдений. Это направление исследований представляет интерес, например, для вспомогательных задач наземно-космической связи, применительно к вспомогательным задачам проектирования перспективных сетей связи. Совокупность этих обстоятельств обусловливает необходимость дополнительного и повторного анализа ситуации для технико-экономической оптимизации аппаратурно-методического обеспечения метода радиометрии.

В работе рассмотрены:

- необходимость учета микроклиматических особенностей местности при проектировании перспективных систем радиорелейной и наземно-космической связи;

- развитие аппаратуры радиометрического измерительного комплекса за счет использования сверхмалошумящих бюджетных приемников СМ ДВ и оригинальных схемных и алгоритмических решений;

- методическое обеспечение микроволновых радиометрических наблюдений с оценкой точности конкурирующих методов калибровки и аппаратурных параметров.

Кроме того, в интересах решения проблем ДЗ и для решения коммуникационных задач рассмотрен выбор оптимальных рабочих частот. Выбор проводится с учетом новых тенденций в развитии элементной базы для высокочувствительных широкополосных приемников. Проектирование и практическая проверка работоспособности созданного трехчастотного радиометрического комплекса проводились с учетом требований комплексного обеспечения необходимой чувствительности радиометров и минимизации их стоимости.

Полученные результаты могут быть полезны при проектировании многочастотных измерительных радиометрических систем, при подготовке и проведении экспериментальных исследований радиофизических параметров объектов окружающей среды. 


\section{1. Постановка задачи}

Актуальность изучения статистических параметров полного вертикального и погонного приземного ослабления радиоволн ММ диапазона резко возросла ввиду разработки перспективных сверхскоростных и информационных сетей наземно-космической и тропосферной связи пятого поколения (5G), использующих частотные диапазоны $10-100$ ГГц. Применение этого относительно нового для широкомасштабно используемых коммуникационных приложений диапазона обеспечивает рост объемов и скорости передаваемой информации выше 10 Гбит/с при приемлемых уровнях готовности в сетях связи $\left(10^{-3}-10^{-4}\right)$.

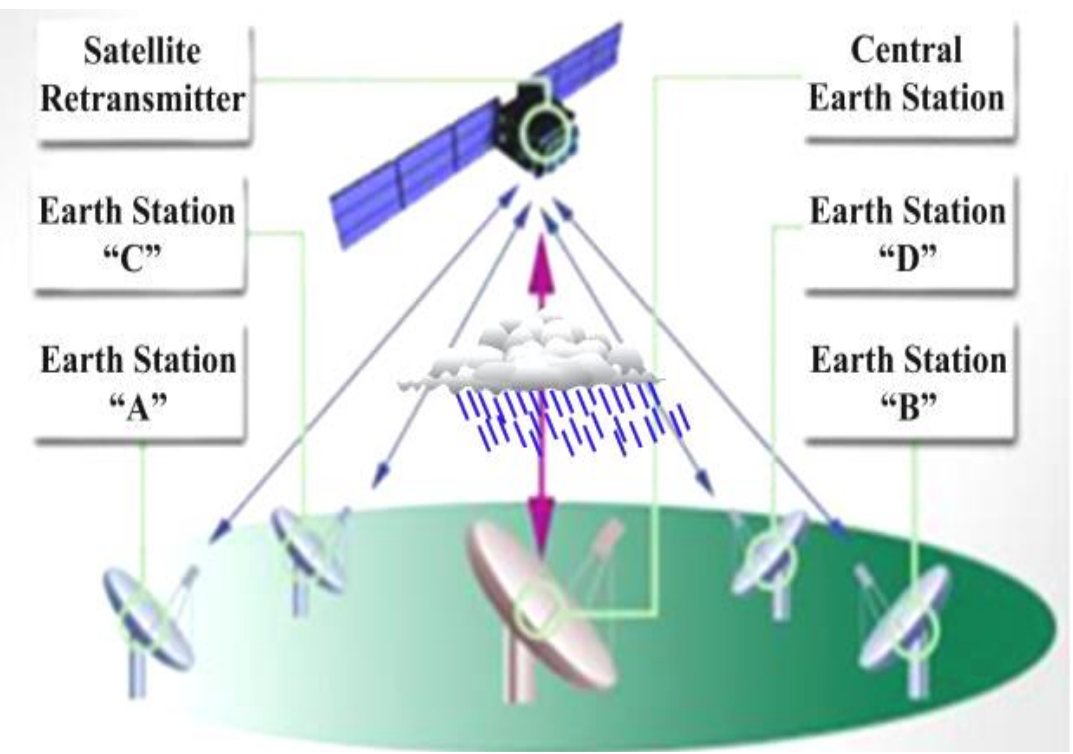

Рис.1. Пример организации сети спутниковой связи типа «Звезда»

При этом величина коэффициента готовности линии связи является ключевой характеристикой, определяющей надежность передаваемой информации. Рассчитать параметр готовности/неготовности линии связи можно, используя рекомендации международного союза по телекоммуникациям ITU $[2,3]$, на основе выражений расчета бюджета радиолинии на трасcе [4]:

$$
\mathrm{P}_{\mathrm{r}}=\mathrm{P}_{\mathrm{t}}+\mathrm{G}_{\mathrm{t}}-\mathrm{L}_{\mathrm{FS}}-\mathrm{L}_{\text {rain }}-\mathrm{L}_{\mathrm{atm}}+\mathrm{G}_{\mathrm{r}} \text {, }
$$

где $\mathrm{P}_{\mathrm{t}}$ - мощность передатчика в $d \mathrm{Bm} ; \mathrm{G}_{\mathrm{t}}, \mathrm{G}_{\mathrm{r}}$ - коэффициент усиления антенны передатчика и приемника в $\mathrm{dBi} ; \mathrm{L}_{\mathrm{FS}}$ - затухание сигнала в свободном пространстве [5]; $\mathrm{c}=3 \cdot 10^{8}-$ скорость света, м/с;

$$
\mathrm{L}_{\mathrm{FS}}=20 \lg \frac{4 \pi \mathrm{df}}{\mathrm{c}}
$$

Затухание сигнала в дожде [4]

$$
\mathrm{L}_{\text {rain }}=\mathrm{L}_{\mathrm{sp} \_ \text {rain }} \cdot \mathrm{r} \cdot \mathrm{d} / 1000
$$

где $\mathrm{L}_{\mathrm{sp} \_ \text {rain }}$ - специфический коэффициент затухания $d B m / \kappa м$ [2]; $\mathrm{d}$ - длина трассы, м; r - поправка;

$$
\mathrm{L}_{\mathrm{sp} \_ \text {rain }}=\mathrm{k}(\mathrm{f}) \cdot \mathrm{RR}^{\alpha(\mathrm{f})} \text {, }
$$

где $\mathrm{RR}$ - интенсивность дождя, мм/ч; $\mathrm{k}(\mathrm{f})$ и $\alpha(\mathrm{f})$ константы, зависящие от частоты и поляризации сигнала [2].

Поправка $\mathrm{r}$, которая учитывает, что дождь падает только на часть линии связи, определяется из выражения [5] 


$$
\mathrm{r}=(1+\mathrm{d} / 35 \exp (-0.015 \mathrm{RR}))^{-1}
$$

Затухание сигнала в атмосферных газах

$$
\mathrm{L}_{\mathrm{atm}}=\mathrm{L}_{\mathrm{sp}_{-} \mathrm{atm}} \cdot \mathrm{d} / 1000,
$$

где $\mathrm{L}_{\mathrm{sp} \_ \text {atm }}$ - специфическое затухание $d B m / \kappa M$, которое вычисляется по рекомендациям ITU [3].

Максимальные потери в дожде могут быть определены из уравнения (1) с использованием чувствительности приемника $\mathrm{P}_{\mathrm{r}_{-} \text {min }}$ для заданного значения битовой ошибки (BER) следующим образом [6]:

$$
\mathrm{L}_{\text {rain_max }}=\mathrm{P}_{\mathrm{t}}+\mathrm{G}_{\mathrm{t}}+\mathrm{G}_{\mathrm{r}}-\mathrm{P}_{\mathrm{r}_{-} \min }-\mathrm{L}_{\mathrm{FS}}-\mathrm{L}_{\mathrm{atm}},
$$

Подставляя (4) - (6) в (8), получаем

$$
\frac{\mathrm{RR}_{\max }^{\alpha(\mathrm{f})}}{1+\mathrm{d} / 35 \exp \left(-0.015 \mathrm{RR}_{\max }\right)}=\frac{1000}{\mathrm{k}(\mathrm{f}) \mathrm{d}}\left(\mathrm{P}_{\mathrm{t}}+\mathrm{G}_{\mathrm{t}}+\mathrm{G}_{\mathrm{r}}-\mathrm{P}_{\mathrm{r}_{-} \min }-\mathrm{L}_{\mathrm{FS}}-\mathrm{L}_{\mathrm{atm}}\right) .
$$

Из уравнения (9) можно определить максимальное значение дождевой нормы RRmax, которое гарантирует доступность канала связи, в зависимости от частоты, расстояния и мощности передатчика. Однако левая часть выражения (9) является очень сложным выражением RRmax, и поэтому для его решения нужно использовать вычислительные методы. Кроме того, если известно статистическое распределение интенсивности дождей $\mathrm{p}(\mathrm{RR})$ (вероятность p, величина интенсивности дождей для которой больше RR), можно получить вероятность нарушения связи.

Пример такого расчета, проведенного для тропосферной трассы в Болгарии с учетом статистики дождей [6], показан на рис. 2.

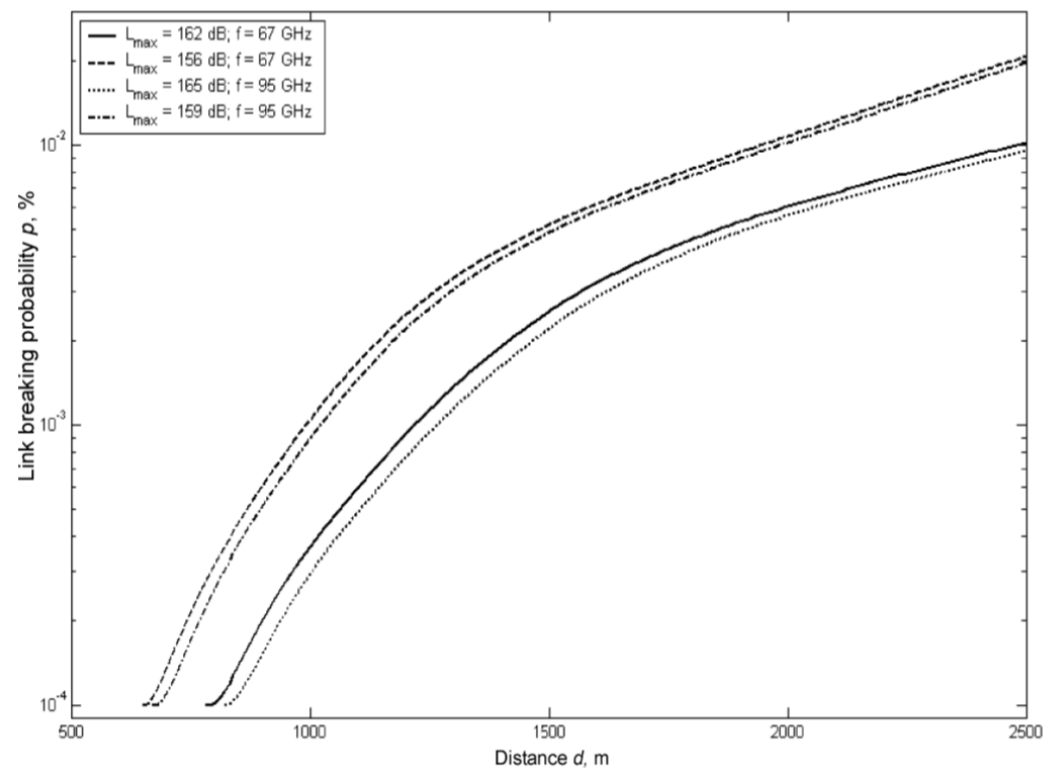

Рис. 2. Вероятность разрыва связи $p(\%)$ в зависимости от длины $d$ горизонтальной трассы для частот 67 и 95 ГГи [6]

Для таких расчетов, помимо конкретизации задаваемых аппаратурных параметров линии связи (чувствительность приемника, излучаемая мощность передатчика, коэффициент усиления антенны и др.), необходимы данные о кумулятивной функции распределения атмосферного ослабления для каждого рассматриваемого региона. При отсутствии такой стати- 
стической информации для некоторых регионов мира, можно воспользоваться предлагаемыми ITU приближенными значениями, характерными для условно разделенных на глобусе зон (рис. 3).

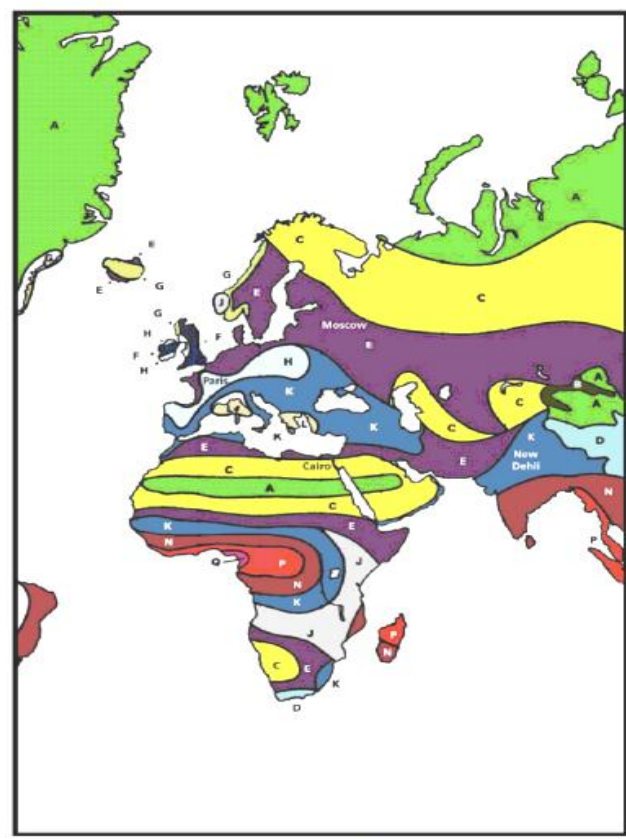

Europe/Africa Rain Regions

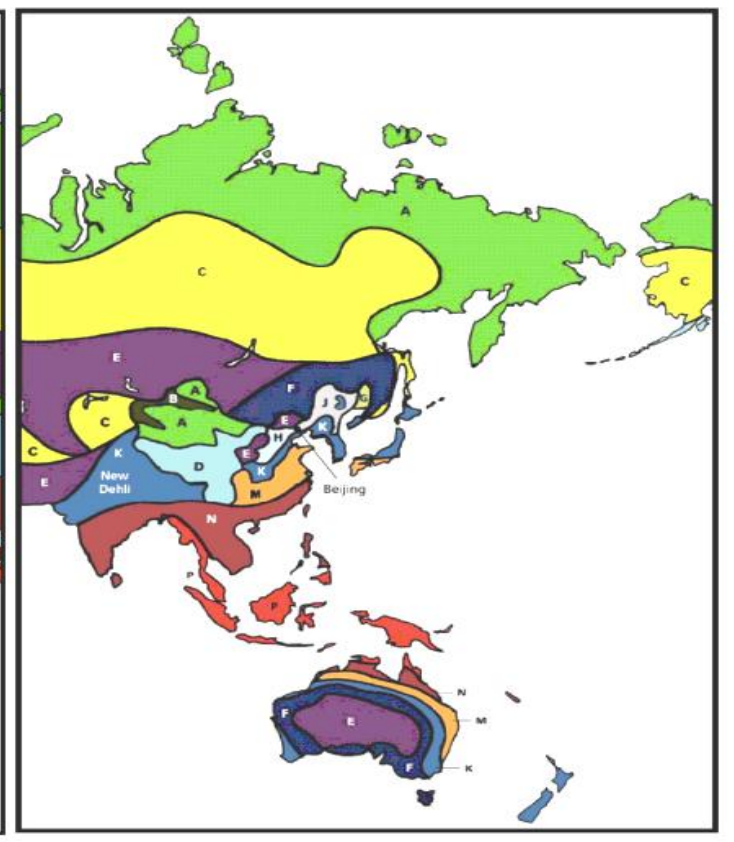

Asia-Pacific Rain Regions

Рис. 3. Укрупненная карта климатических зон по ITU [2]

Ввиду важности получения максимально адекватных оценок прогнозирования надежности связи ITU рекомендует восполнять пробелы модельного учета микроклиматических особенностей каждого региона экспериментальным накоплением сезонной и годовой статистики атмосферного ослабления. Известно, что с увеличением рабочей частоты стремительно уменьшается дальность связи, повышается зависимость прохождения сигнала, а значит и зависимость пространственной конфигурации и размера сетевой соты, от погодных и микроклиматических условий, характерных для конкретного региона.

Для территории Украины такие экспериментальные данные о кумулятивных функциях вертикального и горизонтального атмосферного ослабления в ММ ДВ отсутствуют, притом, что эта информация важна для экономической эффективности принятия технических решений и для конкурентной способности их практического внедрения. Некоторым исключением можно считать приближенные оценки [7], сделанные на основе многолетних метеоданных, полученных с более чем двадцатиминутным интервалом времени выборки измерений. Несмотря на этот существенный недостаток, который ограничивает надежность полученных абсолютных значений кумулятивной функции (желательно использовать интервал выборки не более 1 мин), обращает внимание многократное различие в вероятности событий с повышенным ослаблением при сравнении некоторых регионов Украины (рис. 4). Эта выявленная качественная закономерность лишь подтверждает необходимость дополнительных исследований для учета микроклиматических особенностей местности для инфраструктурной и аппаратурной оптимизации проектируемых перспективных наземно-космических и приземных средств и сетей связи.

Важной составляющей при подготовке и проведении исследований является используемое аппаратурно-методическое обеспечение.

На горизонтальных трассах накопление недостающих данных может быть проведено на основе приемной и передающей аппаратуры, установленной на приземных трассах, в совокупности с вспомогательной метеорологической аппаратурой с минутным интервалом измерений. 


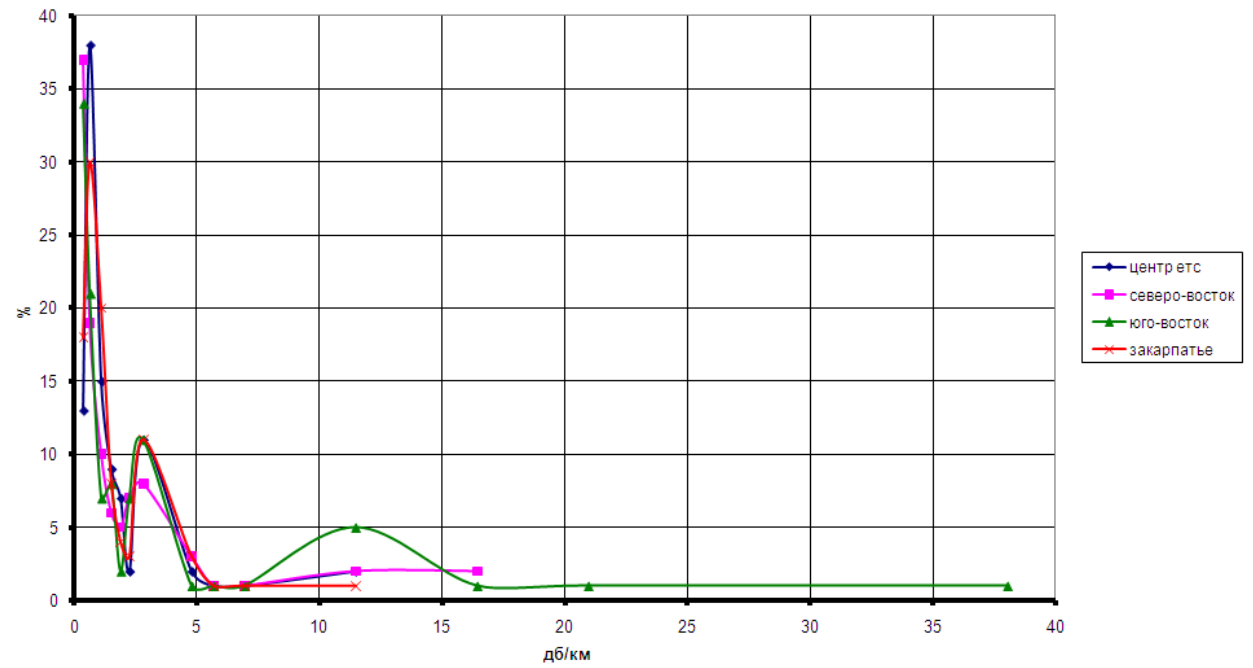

Рис. 4. Гистограмма вероятности распределения погонного приземного атмосферного ослабления $\gamma$ в диапазоне 94 ГГц (август) для четырех регионов Украины [7]

На вертикальных трассах наиболее удобны аппаратура и методы радиометрии, использование которых более характерно для задач ДЗ окружающей среды. Эти направления исследований можно проводить с использованием одного и того же оборудования. Желательно, чтобы оборудование позволяло измерять и анализировать значения полного вертикального ослабления радиоволн в нескольких частотных диапазонах одновременно.

\section{2. Выбор рабочих частот и схема построения измерительного комплекса}

\section{1. Обоснование выбора рабочих частот}

Процедура определения физических параметров атмосферы, как правило, состоит из следующих этапов получения и обработки данных:

а) измерение антенной температуры радиометра, которую можно представить как:

$$
\mathrm{T}_{\mathrm{a}}=\mathrm{T}_{\text {eff }}\left(1-\mathrm{e}^{\tau \sec \theta}\right)(1-\beta) \mu+\mathrm{T}_{\mathrm{bgr}} \beta \mu+2.7 \mathrm{e}^{-\tau \sec \theta}(1-\beta) \mu+\mathrm{T}_{\mathrm{o}}(1-\mu)
$$

где $\mathrm{T}_{\mathrm{bgr}}$ - усредненная величина радиояркостной температуры фона принимаемой вне основного лепестка диаграммы направленности (ДН) антенны радиометра; $\beta$ - коэффициент рассеяния вне основного лепестка ДН антенны; $\mu$ - к.п.д. антенны;

б) определение радиояркостной температуры атмосферы $\mathrm{T}_{\mathrm{b}}$ в направлении главного лепестка ДН антенны по измеренным значениям $\mathrm{T}_{\mathrm{a}}$ с учетом выражений (1) и (10);

в) определение значений $\tau$ (непер) и полного вертикального ослабления (дБ) на основе данных о $\mathrm{T}_{\mathrm{b}}$ с целью построения кумулятивных функций распределения этих параметров для телекоммуникационных приложений. При решении задач ДЗ данные о значениях $\tau$ на разных частотах необходимы для решения системы уравнений:

$$
\alpha_{\text {total }}^{\lambda_{\mathrm{i}}}=\alpha_{\text {oxigen }}^{\lambda_{\mathrm{i}}}+\Phi_{\lambda_{\mathrm{i}}} \mathrm{Q}+\mathrm{k}_{\lambda_{\mathrm{i}}}\left(\mathrm{T}_{0}\right) \mathrm{W}
$$

где i - номер частотного диапазона, Q - восстанавливаемое значение влагозапаса атмосферы (парообразная влага); W - восстанавливаемое значение водозапаса атмосферы (капельная влага); $\Phi_{\lambda_{\mathrm{i}}}$ и $\mathrm{k}_{\lambda_{\mathrm{i}}}\left(\mathrm{T}_{0}\right)$ - коэффициенты удельного поглощения в парообразной и жидкокапельной влаге атмосферы соответственно.

Понятно, что решение таких систем уравнений с двумя переменными $\mathrm{Q}$ и $\mathrm{W}$ более предпочтительно для случаев существенного различия используемых частот.

Для определения наиболее предпочтительной пары частот для таких приложений ДЗ нами рассчитаны коэффициенты линейного поглощения в облаках (дБҺГ/ $\mathrm{m}^{2}$ ) в зависимости 
от их температуры для частот 4, 12, 20, 40 и 94 ГГц (табл. 1). Для расчета этих данных использовалась известная модель МРМ (Atmospheric Millimeter Waves Propagation Model) [8]. Уместно заметить, что каждая из рассмотренных частот полезна не только для решения задач Д3, но и для разработки перспективных средств связи, например в формате 5G.

Таблица 1

\begin{tabular}{|c|c|c|c|c|c|c|c|}
\hline \multirow{2}{*}{ Частота, ГГц } & \multicolumn{6}{|c|}{ Температура, ${ }^{\circ} \mathrm{C}$} & \multirow{2}{*}{ 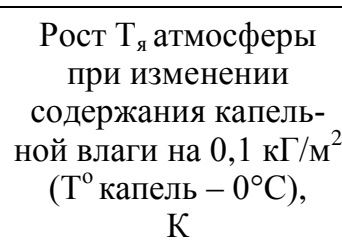 } \\
\hline & -30 & -20 & -10 & 0 & 10 & 20 & \\
\hline 4 & 0.039 & 0.031 & 0.021 & 0.015 & 0.011 & 0.009 & 0,1 \\
\hline 12 & 0.327 & 0.269 & 0.188 & 0.133 & 0.098 & 0.077 & 0,9 \\
\hline 19 & 0.733 & 0.625 & 0.454 & 0.326 & 0.244 & 0.191 & 2,1 \\
\hline 41 & 2.111 & 2.029 & 1.732 & 1.363 & 1.067 & 0.857 & 9 \\
\hline 94 & 4.243 & 4.457 & 4.824 & 4.73 & 4.284 & 3.757 & 31 \\
\hline
\end{tabular}

Частоты 40 и 94 ГГц считаются традиционными в задачах ДЗ атмосферы. Включение в рассмотрение частот 4, 12, 20 ГГц связано с возможностью построить в этих диапазонах высокочувствительных и в то же время высокобюджетных радиометров на основе широко используемых современных промышленных приемников спутниковых телевизионных сигналов с показателем шума менее 1 дБ. Расчеты показали, что использование таких приемников при построении радиометра позволяет обеспечить достижение флуктуационной чувствительности в несколько сотых, а возможно и тысячных долей К.

Анализ данных табл. 1 показывает, что наиболее чувствительными к изменению водозапаса облачного покрытия являются радиометры диапазона 94 ГГц. При этом реакция радиометра диапазона 4 ГГц на изменения водозапаса облаков будет более чем на два порядка меньшей, чем в диапазоне 94 ГГц. Это обстоятельство вынуждает исключить возможность применения С ДВ из дальнейшего рассмотрения в качестве второго дополнительного канала. Реакция радиометра диапазона 12 ГГц примерно в 30 раз отличается от радиометров, работающих в диапазоне 94 ГГц. Однако, на наш взгляд, его использование может быть оправдано тем, что в этом диапазоне волн можно реализовывать примерно в 30 раз большую чувствительность, а также тем, что такое устройство требует наименьших финансовых затрат для его реализации. Радиометры, построенные в других рассматриваемых ДВ, будут иметь промежуточные показатели по рассматриваемым параметрам стоимости, чувствительности и реакции на изменение атмосферной капельной влаги.

Обращает на себя внимание также то, что температурная зависимость коэффициентов погонного поглощения в каплях существенна для всех рассматриваемых частот, за исключением 94 ГГц. Это указывает на необходимость достаточно точного знания эффективной температуры облачного покрытия при решении задач восстановления его водозапаса.

В табл. 2 приведены полученные расчетные значения (в разах) соотношений между величинами погонного поглощения облаков (дБ/км) для разных температур капель и для разных пар частот.

Таблица 2

\begin{tabular}{|c|c|c|c|c|c|c|c|}
\hline \multirow{2}{*}{ Частота, ГГц } & \multicolumn{7}{|c|}{ Температура, ${ }^{\circ} \mathrm{C}$} \\
\cline { 2 - 8 } & -30 & -20 & -10 & 0 & 10 & 20 & \\
\hline $94 / 12$ & $\mathbf{1 3}$ & $\mathbf{1 6 , 5 6}$ & $\mathbf{2 5 , 5 6}$ & $\mathbf{3 5 , 5 6}$ & $\mathbf{4 3 , 7}$ & $\mathbf{4 8 , 8}$ & max \\
\hline $94 / 19$ & 5,8 & 7,2 & 10,6 & $14,5$. & 17,6 & 19,7 & \\
\hline $94 / 41$ & 2,0 & 2,2 & 2,8 & 3,5 & 4,0 & 4,4 & \\
\hline $41 / 12$ & 6,4 & 7,5 & 9,2 & 10,25 & 10,9 & 11,1 & \\
\hline $12 / 4$ & 8,4 & 8,7 & 9,0 & 8,9 & 8,9 & 8,6 & \\
\hline $19 / 12$ & $\mathbf{2 , 2 5}$ & $\mathbf{2 , 3}$ & $\mathbf{2 , 4}$ & $\mathbf{2 , 4 5}$ & $\mathbf{2 , 4 9}$ & $\mathbf{2 , 4 8}$ & min \\
\hline
\end{tabular}


Видно, что если для одних пар частот температурная зависимость соотношений отсутствует, либо выражена слабо, то для пар частот 94ГГц/19ГГц, и особенно для 94ГГц/12ГГц, такая зависимость ярко выражена. Это обстоятельство можно использовать для мониторинга эффективной температуры облаков при восстановлении параметров водозапаса по данным двухчастотных радиометрических измерений.

На данном этапе была выбрана пара частот 12 ГГц и 94 ГГц для реализации измерительной системы, предназначенной для дистанционного мониторинга атмосферной влаги.

Помимо ясной или облачной атмосферы в практике дистанционного зондирования, а также в современных и перспективных системах связи важную и требующую учета роль играют дожди $[6,9]$. Расчеты частотной зависимости погонного поглощения в дождях различной интенсивности I, а также зависимости $\gamma$ от интенсивности для выделенных выше пяти частот были проведены с помощью модели Лииба [8]. В табл. 3 приведен коэффициент удельного ослабления в дожде (дБҺкГ/м ${ }^{2}$ ) в зависимости от их температуры для частот 4,12 , 20, 40 и 94 ГГц.

Полученные данные указывают также на бесперспективность использования С ДВ (4 ГГц) для дистанционного мониторинга параметров дождей методом радиометрии из-за очень малого влияния дождевых капель на величину погонного поглощения атмосферой в этом ДВ.

Таблица 3

\begin{tabular}{|c|c|c|c|c|c|c|c|c|c|c|}
\hline \multirow{2}{*}{$\begin{array}{c}\text { Частота, } \\
\text { ГГц }\end{array}$} & \multicolumn{9}{|c|}{ Интенсивность дождя (мм/ч) } & \\
\hline & 1 & 5 & 10 & 15 & $\begin{array}{l}\text { Соотношение } \gamma \\
\text { (для } 1 \text { и } 15 \mathrm{Mm} / ч)\end{array}$ & 20 & 40 & 80 & 120 & \\
\hline 94 & 0,98 & 3,35 & 6,0 & 7,77 & $\sim 8$ & 9,7 & 16,4 & 28,0 & 38,0 & дБ/Км \\
\hline 40 & 0,32 & 1,5 & 2,9 & 4,3 & $\sim 14$ & 5,7 & 11,1 & 21,7 & 32,0 & дБ/Км \\
\hline 20 & 0,06 & 0,36 & 0,77 & 1,2 & $\sim 20$ & 1,7 & 3,60 & 7,90 & 12,4 & дБ/км \\
\hline 12 & 0,017 & 0,112 & 0,25 & 0,4 & $\sim 25$ & 0,56 & 1,25 & 2,80 & 4,48 & дБ/Км \\
\hline 4 & 0,0012 & 0,0066 & 0,014 & 0,021 & $\sim 18$ & 0,029 & 0,06 & 0,125 & 0,20 & дБ/км \\
\hline
\end{tabular}

В то же время видно, что кратность увеличения атмосферного затухания, вызванного ростом интенсивности дождя в СМ ДВ в 2-3 раза выше, чем наблюдаемый рост этого параметра в ММ ДВ. При этом абсолютные значения погонного поглощения в диапазоне 20 ГГц можно считать приемлемыми для их измерения радиометрическим методом и для последующего восстановления высоты дождевого слоя по данным трехчастотных измерений. В перспективе предполагается развитие описанной ниже измерительной системы путем введения радиометрического канала диапазона 20 ГГц в дополнение к уже созданным каналам. Это решение должно расширить возможности атмосферного мониторинга, обеспечив восстановление такого дополнительного параметра, как высота дождевого слоя.

\section{2. Схема построения измерительного комплекса}

Структурная схема радиометрического двухчастотного комплекса Национального аэрокосмического университета «ХАИ» (ХАИ) и схема измерений показаны на рис. 5.

Схема содержит следующие блоки: $\mathrm{A}$ - соответствующая антенна $(\mathrm{i}=1,2) ; \mathrm{AP}_{\mathrm{i}}$ - антенный луч; LNA - усилитель с низким уровнем шума; LPA - фильтр нижних частот; PIN DM это ріп-диодный модулятор; FC - ферритовый циркулятор; M - смеситель; BPF - полосовой проходной фильтр; GO - генератор Ганна; CG - управляющий генератор; IFA - усилитель промежуточной частоты; SqD - квадратный детектор; SD - синхронный детектор; DCA усилитель постоянного тока; ADC - аналого-цифровой преобразователь. 


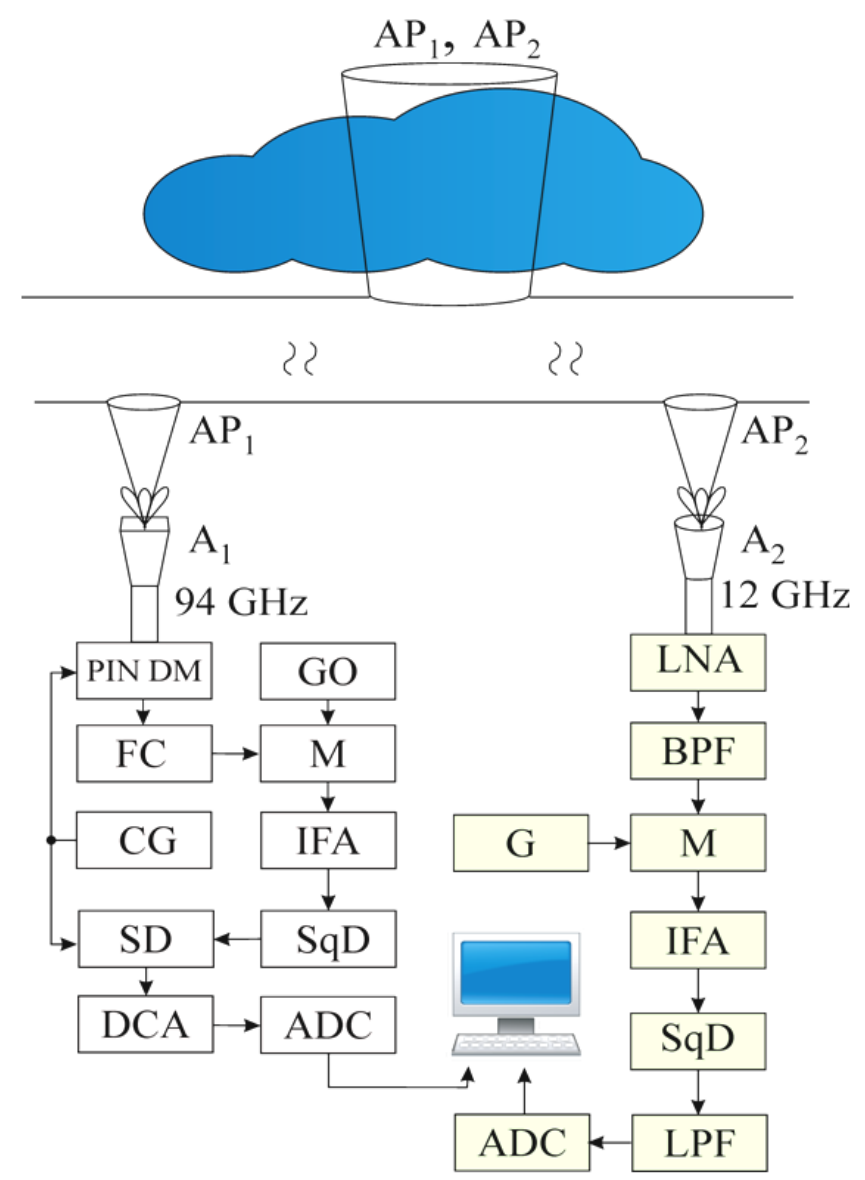

Рис. 5. Блок-схема радиометрического комплекса и схемы измерения

Видно, что оба радиометра реализованы по разным схемам. Радиометр, работающий на антенну $\mathrm{A}_{1}$, - это бездиплексерный радиометр модуляционного типа [10]. Центральная частота настройки приемника 94 ГГц и ширина полосы частот по входу 600 МГц. Работа этого радиометра, обеспечивающего флуктуационную чувствительность радиотеплового сигнала $0.25 \mathrm{~K}$, изложена в [11].

Второй канал радиометра реализован по схеме радиометра полной мощности с однократным преобразованием частоты. Этот радиометр полной мощности 3 см ДВ построен на основе типовой платы ТВ конвертора супергетеродинного типа Х-диапазона.

Прием сигнала шумового сигнала осуществляется в однополосном режиме в диапазоне 11.7 - 12.75 ГГц. На входе радиометрического приемника используется полевой транзистор типа NE4210S01-T1 (NEC) с коэффициентом шума не более 0.75дБ. С выхода приемника шумовой сигнал поступает на квадратичный детектор, цепь интегратора, УПТ и АЦП. Измеренная флуктуационная чувствительность радиометра, работавшего в режиме приема полной мощности шумового сигнала, составила $0.03 \mathrm{~K}$.

Радиометрический комплекс 3 см и 3 мм ХАИ показан на рис. 6. Антенна 12 ГГц показана слева на рис. 6, а справа показана антенна 94 ГГц. Все блоки комплекса расположены в коробке под антеннами.

Измерительная система Харьковского национального университета радиоэлектроники (ХНУРЭ) 8 мм ДВ с флуктуационной чувствительностью 0,2 К показана на рис. 7. Схема построения и состав этого радиометра описаны в [12].

Оцифрованные сигналы с выходов АЦП передаются на персональный компьютер для последующих расчетов, обработки и представления данных. 


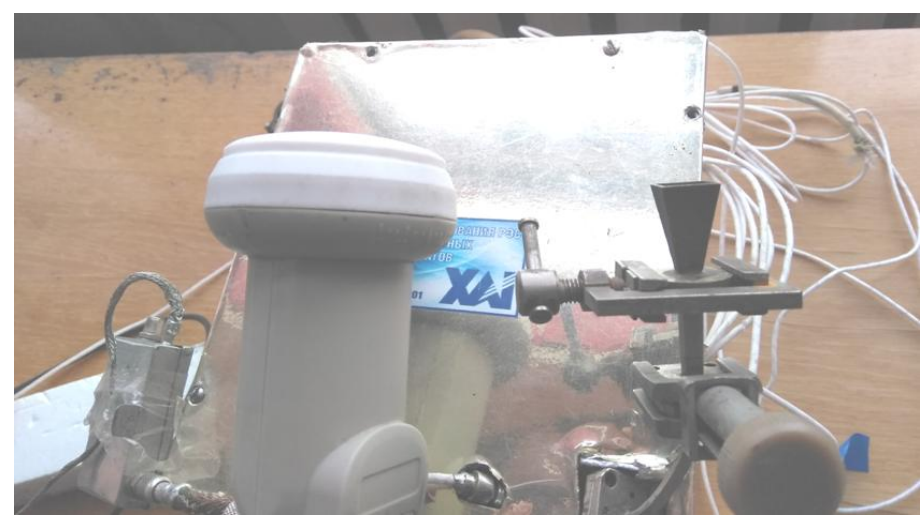

Рис. 6. Радиометрический комплекс 3 см и 3 мм ХАИ

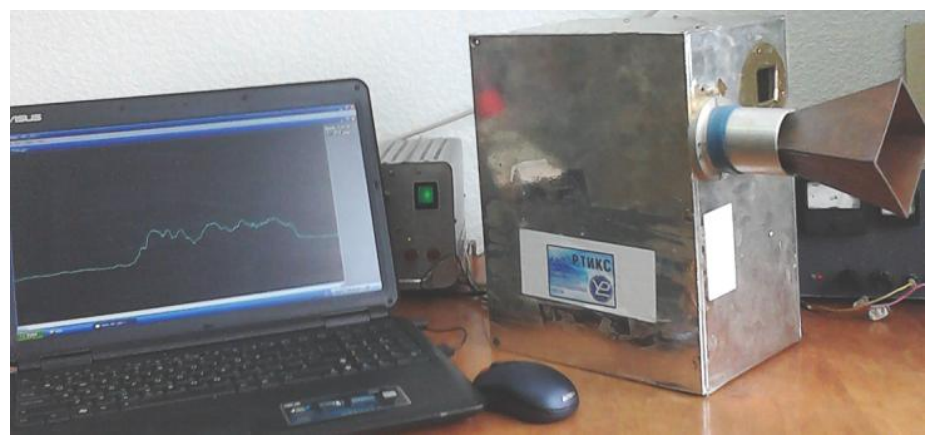

Рис. 7. Радиометрический комплекс 8 мм ХНУРЭ

Совместное использование трехчастотного радиометрического комплекса предполагается для задач ДЗ атмосферной влаги и параметров облаков и для исследований изменчивости значений полного вертикального ослабления в задачах наземно-космической связи [13], в том числе линий связи 5G. Для реализации коммуникационной составляющей этой программы атмосферных исследований в интересах построения перспективных беспроводных каналов связи создана экспериментальная приземная линия связи (рис. 8, a) на основе использования точек доступа MikroTik Wireless Wire (RBwAPG-60ad kit) в диапазоне 60 ГГц (рис. 8, б).

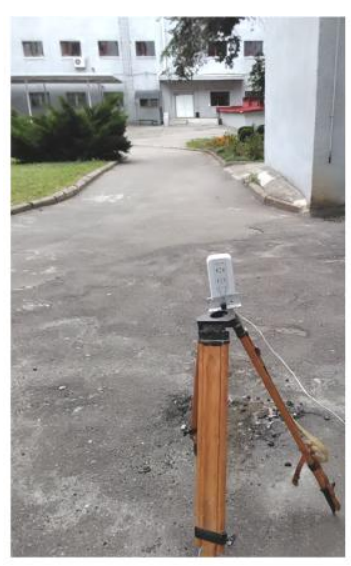

$a$

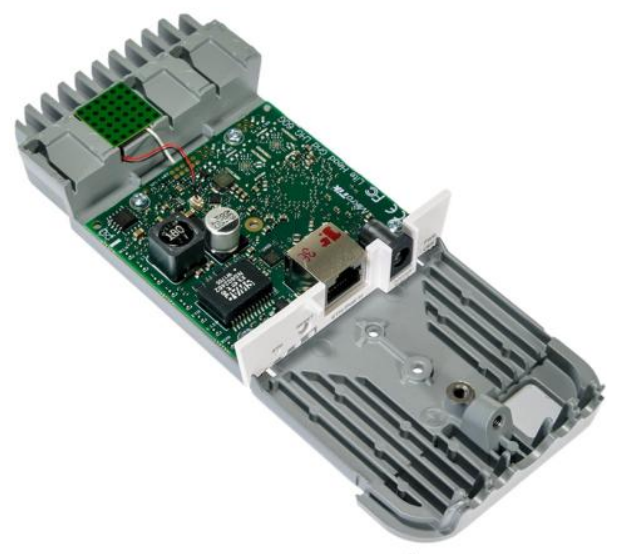

6

Рис. 8. Экспериментальная линиясвязи (a) диапазона 60 ГГц на основе радиомодемов MikroTik Wireless Wire (б)

В этой системе связи используется фазированная антенная решетка для формирования узкой диаграммы направленности и увеличения энергетического потенциала линии связи.

\section{3. Результаты экспериментальных исследований}

С помощью описанного радиометрического комплекса проведены различные предварительные экспериментальные исследования. 
Каждый из радиометрических каналов независимо испытан для анализа атмосферных образований (облаков). На рис. 9 фрагменты изменения яркости неба во времени регистрируются на длине волны 3 см (апрель 2017 г.) и 3 мм (май 2017 г.) при прохождении двух весенних атмосферных фронтов над пунктом измерения в течение 25 и 16 часов соответственно.
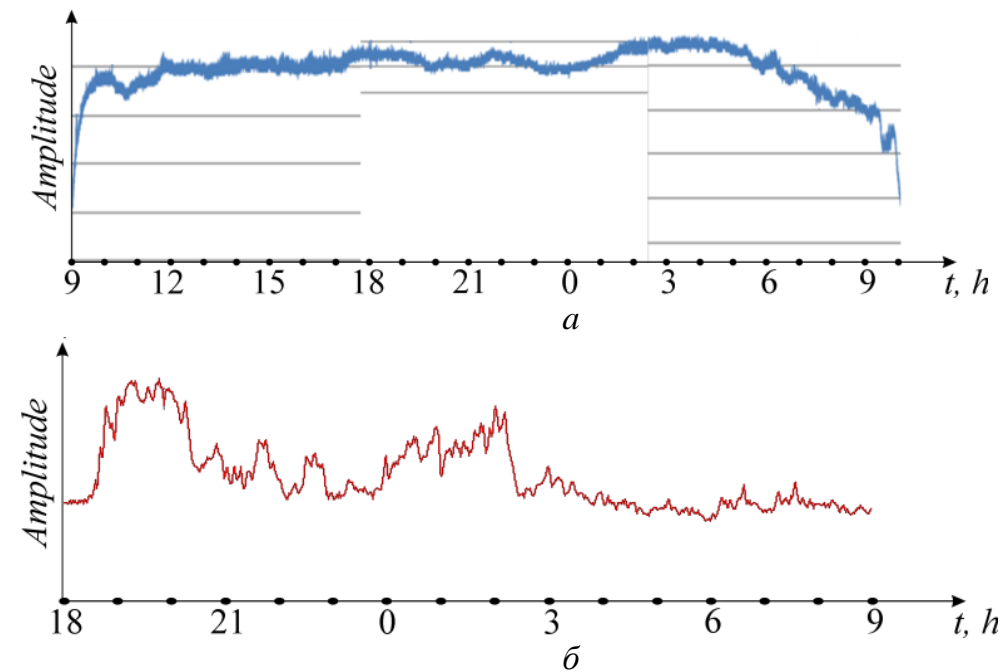

Рис. 9. Фрагменты записи изменения радиояркостной температуры неба в 3см ДВ ( $a$ - апрель 2017) и 3 мм ДВ ( $\sigma$ - май) при прохождении двух весенних атмосферных фронтов над измерительным пунктом в течение 25 и 16 часов соответственно

Разработанный прототип измерительного комплекса успешно прошел лабораторные и натурные испытания. На рис. 10 показан фрагмент записи изменения радиоизлучения неба в 3 и 8 мм ВБ при прохождении фронтальной зоны облачности. Приведенные на рисунке данные позволяют восстановить динамику изменения полного вертикального ослабления в атмосфере, значений эффективной температуры конденсированной влаги в небе, значений содержания капельной воды и парообразной влаги в облаках.

Результат восстановления эффективной температуры облачного слоя по данным изменений радиоизлучения неба, изображенных на рис. 10, показан на рис. 11. Использованная процедура восстановления эффективной температуры облачного слоя основана на хорошо известных формулах двойного Дебая [8].

Полученные значения содержания пара в атмосфере Q и жидкости W в облаках представлены на рис. 12, $a, 6$.

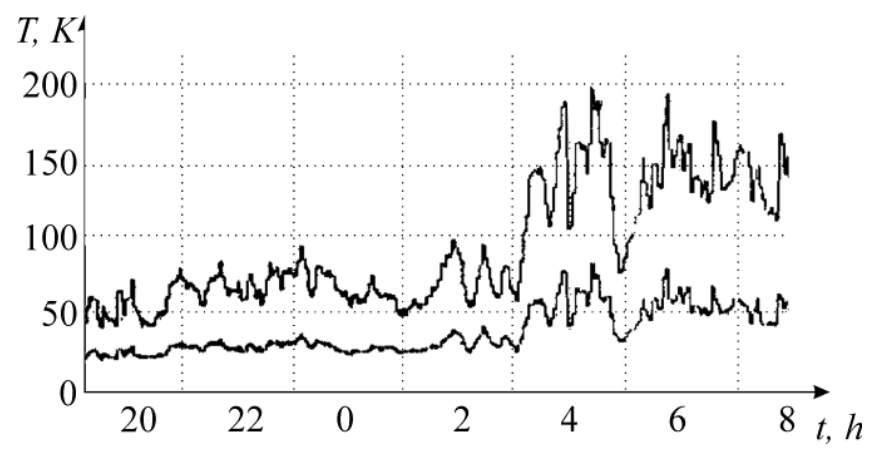

Рис. 10. Фрагмент записи изменения радиоизлучения неба на частоте 94 ГГц (верхняя кривая) и 40 ГГц при прохождении через фронтальную зону облачности с 19 до 8 часов утра 


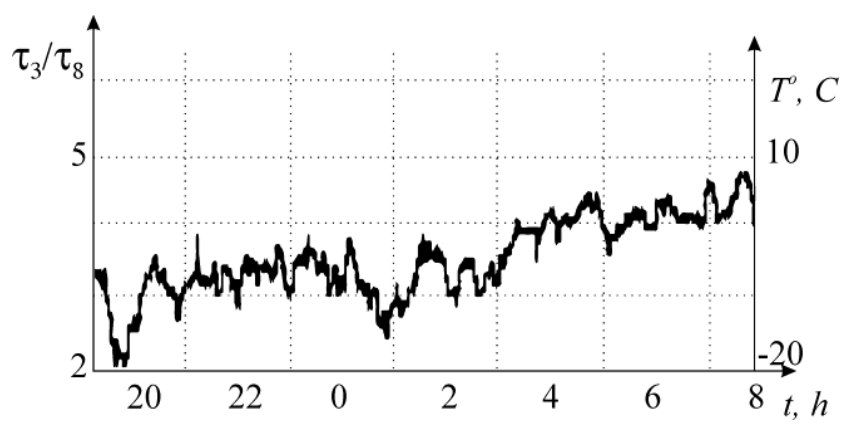

Рис. 11. Результат восстановления эффективной температуры облачного слоя
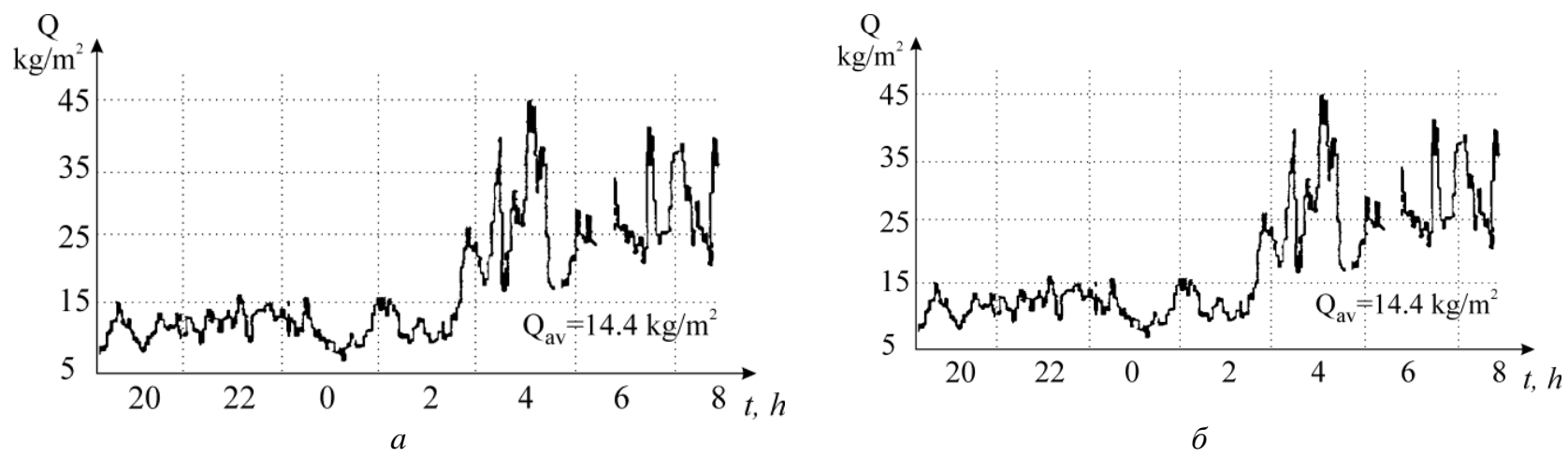

Рис. 12. Восстановленные значения влаго-( $a)$ и водо- (б) содержания облаков

Анализ первичных данных двуволновых радиометрических измерений в 3 и 8 мм ДВ позволяет определять зоны облаков, которые содержат кристальную влагу, а поляризационный режим измерений дает возможность исследовать морфологическую структуру кристаллов замороженной влаги.

Возможности обнаружения человеческого тела с помощью трехсантиметрового радиометра и трехмиллиметрового радиометра на трех разных фонах показаны на рис. 13, $a, \sigma$ соответственно. Здесь показана реакция выходных сигналов радиометров на пересечение их диаграмм, которые направлены в сторону окна под углом 20 градусов над горизонтом. Первый уровень сигнала (см. стрелки 1 на рис. 13) получен при открытии обеих оконных рам, второй (см. стрелки 2 на рис. 13) - когда одна оконная рама была закрыта, третий (см. стрелки 3 на рис. 13) - когда обе оконные рамы были закрыты. Одиночные всплески на графиках соответствуют моментам пересечения человеком диаграмм направленности радиометрического комплекса.

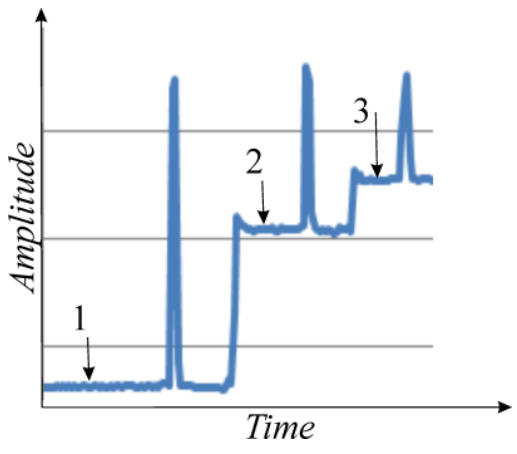

$a$

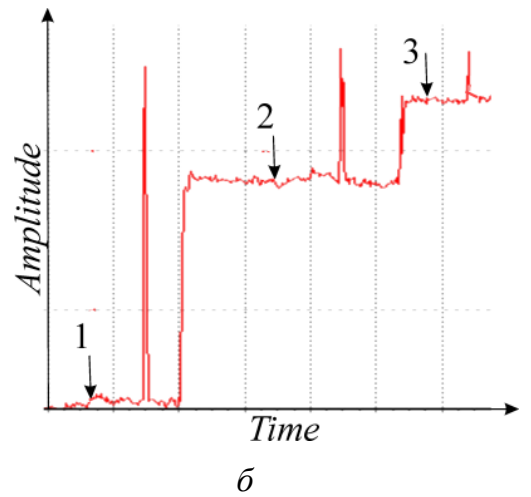

б

Рис. 13. Обнаружение человеческого тела с помощью

трехсантиметрового радиометра ( $a$ ) и 3 мм радиометра (б) на трех разных фонах

Единичные всплески на графиках соответствуют моментам пересечения человеком диаграмм радиометрических антенн.

Оценка пропускной способности экспериментального канала связи 60 ГГц на оборудовании MicroTik в режиме беспроводного моста с использованием программного трафик- 
генератора (рис. 14) показала, что максимальная скорость передачи информации 1 Гбит/с достигается на расстоянии более 120 м в условиях городской застройки [14]. Это подтверждает реализуемость сценария развертывания сетей $5 \mathrm{G}$ в диапазоне 60 ГГц на основе малых сот (Small Cell).

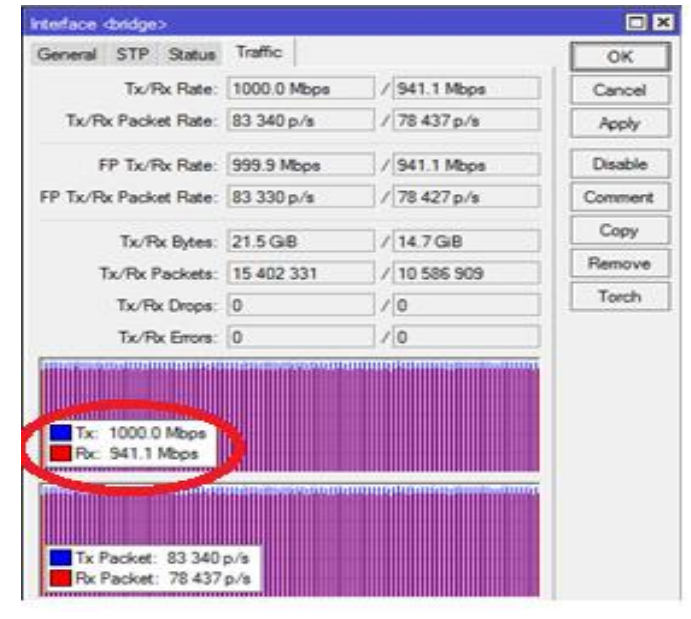

$a$

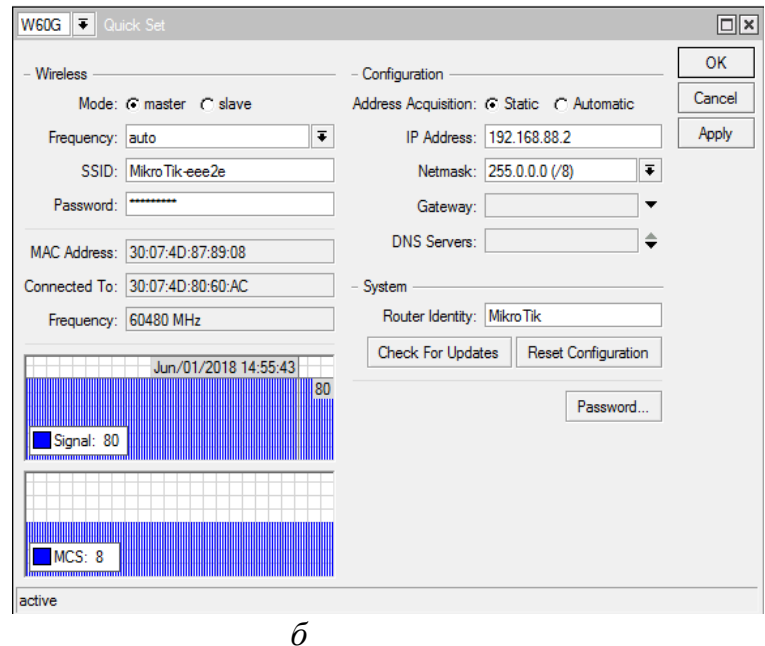

$\sigma$

Рис. 14. Интерфейс программы трафик-генератора, показывающий скорость передачи информации $(a)$, а также уровень сигнала и тип модуляции (б)

\section{4. Методическое обеспечение наблюдений}

В радиометрии интенсивность принятого от исследуемой среды радиотеплового излучения принято выражать в значениях радиояркостной температуры $T_{b}$, представляемой в градусах Кельвина (К). Эта задача решается калибровкой измерительной аппаратуры. Процедура радиометрического мониторинга атмосферы предполагает, помимо обеспечения калибровки измерительной аппаратуры, последующий переход от измеренных значений антенной температуры радиометров $T_{a}$ к радиояркостной температуре объекта излучения [15]. Затем эти данные используются для восстановления значений атмосферного поглощения, а в ряде задач дистанционного зондирования - для последующего восстановления физических параметров наблюдаемых объектов.

К настоящему времени разработаны различные способы решения этих задач. Конкретный метод калибровки радиометрической аппаратуры и методов восстановления параметров атмосферы выбирается, как правило, на основе комплексного и компромиссного рассмотрения требований к точности определяемых параметров, к простоте и удобству процедур измерений. Окончательное решение этого вопроса обусловлено техническими возможностями и наличием вспомогательных устройств и оснастки в условиях лабораторных либо полевых (экспедиционных) измерений.

\section{1. Оценки точности процедур калибровки радиометра}

Калибровка радиометрической аппаратуры проводится для преобразования регистрируемых на выходе радиометра значений напряжения (тока) в значения антенной температуры.

Подавляющее большинство используемых на практике процедур калибровки основано на методе двух согласованных нагрузок антенны, каждая из которых имеет известную и существенно отличную от другой $T_{b}$. При этом одна из согласованных нагрузок антенны радиометра имеет контролируемую температуру окружающей среды. Различие же методов калибровки обычно связано с методом обеспечения контролируемой $T_{b}$ сигнала от второй нагрузки или объекта собственного радиотеплового излучения в используемом ДВ. Тогда величина антенной температуры исследуемого объекта определяется экспериментально как 


$$
\mathrm{T}_{\mathrm{a}}=\mathrm{T}_{0} \frac{\mathrm{T}_{\mathrm{b1}}-\mathrm{T}_{\mathrm{b} 2}}{\mathrm{U}_{1}-\mathrm{U}_{2}}\left(\mathrm{U}_{1}-\mathrm{U}_{\mathrm{x}}\right),
$$

где первый сомножитель представляет собой калибровочный коэффициент $\alpha$ (К/Вольт), $\mathrm{T}_{\mathrm{o}}$ - термодинамическая температура первой нагрузки, $\mathrm{K} ; \mathrm{U}_{1}, \mathrm{U}_{2}, \mathrm{U}_{\mathrm{x}}-$ соответственно уровень выходного сигнала радиометра, принятого от 1-й, 2-й калибровочных нагрузок и от наблюдаемого (исследуемого) объекта В.

В работе проанализировано влияние точности калибровки на определение $\mathrm{T}_{\mathrm{b}}$ неба для методов калибровки, проводимых:

a) с помощью согласованной нагрузки облучателя, находящейся при комнатной температуре (около $300 \mathrm{~K}$ ) и при температуре кипения жидкого азота (около $78 \mathrm{~K}$ );

б) по радиоизлучению неохлаждаемой нагрузки облучателя и радиоизлучению атмосферы (с использованием величины $T_{b}$ атмосферы, рассчитанной либо по метеоданным, либо измеренной с помощью эталонного рупора);

в) по разности сигналов, принятых из зенитного направления ясного неба, и согласованной нагрузки антенны с последующим суммированием этой разности с разностным сигналом между направлениями $60^{\circ}$ от зенита и направлением в зенит. В этом случае указанная сумма сигналов будет находиться в хорошем соответствии с известной величиной термодинамической температуры нагрузки. Это утверждение связано с тем, что радиотепловое излучение неба в направлении $60^{\circ}$ от зенита с высокой степенью точности составляет величину равную $2 \mathrm{~T}_{\mathrm{b}}$ зенита.

Можно также показать, что связь определяемой экспериментально с помощью выражения (12) величины $\mathrm{T}_{\mathrm{a}} \mathrm{c}$ искомой величиной $\mathrm{T}_{\mathrm{b}}$ нисходящего излучения атмосферы можно записать как

$$
\mathrm{T}_{\mathrm{a}}=\mathrm{T}_{\mathrm{b}}(1-\beta) \mu+\mathrm{T}_{\mathrm{bgr}} \beta \mu+2.7 \mathrm{e}^{-\tau \sec \theta}(1-\beta) \mu+\mathrm{T}_{\mathrm{o}}(1-\mu),
$$

где $\mathrm{T}_{\mathrm{b}}=\mathrm{T}_{\mathrm{eff}}\left(1-\mathrm{e}^{\tau \sec \theta}\right)$.

$\mathrm{T}_{\mathrm{b}}$ атмосферы по результатам измерения $\mathrm{T}_{\mathrm{a}}$, как правило, определяется путем использования выражений (10) и (13).

Из анализа этих выражений следует, что кроме калибровочного коэффициента $\alpha$ наихудшее влияние на точность определения $\mathrm{T}_{\mathrm{b}}$ будет оказывать точность определения параметра, характеризующего рассеивающие свойства антенны $p=(1-\beta) \mu$, а также точность оценки усредненных значений фонового излучения $\mathrm{T}_{\mathrm{bgr}}$, принятого из направлений вне основного лепестка ДН антенны. Ввиду простоты обеспечения высокой точности оценки или контроля других параметров, прямо или косвенно входящих в эти выражения (угол визирования, термодинамическая температура окружающей среды $\mathrm{T}_{\mathrm{o}}$ at и др.), в последующем обсуждении мы рассматривать их не будем.

Необходимость определять величину $\mathrm{T}_{\mathrm{bgr}}$ можно исключить из рассмотрения с помощью метода ИЛ. В этом случае $\mathrm{T}_{\mathrm{a}}$ атмосферы $\left(\mathrm{T}_{\mathrm{a} \text { атм }}\right)$ и $\mathrm{T}_{\mathrm{a}}$ ИЛ $\left(\mathrm{T}_{\mathrm{a} \text { ат }}\right)$ измеряется с помощью выбранного метода калибровки, а затем вычисляется $\mathrm{T}_{\mathrm{b}}$ из системы двух уравнений, - выражения (10) и аналогичного ему, записанного для случая, когда диск ИЛ перекрывает главный луч ДН антенны радиометра.

На первом шаге $\tau$ может быть оценено приблизительно, например, рассчитано по метеоданным, ввиду на порядок меньшей величины этого слагаемого. Затем более точное значение $\tau$ можно восстановить, используя значения $\mathrm{T}_{\mathrm{b}}$, определяемые из выражения (13). При таком подходе наиболее существенное влияние на точность измерений радиояркости объекта исследований окажут выбранный метод калибровки радиометрической системы и реализация ее процедуры, а также способ определения параметров рассеяния антенны - p.

Стоит отметить, что восстановление значений полного вертикального поглощения в атмосфере можно проводить разными методами. Например, радиометрическим методом угломестных разрезов [1] или методом просвечивания атмосферы излучаемыми сигналами спутника либо мощного источника космического излучения. Недостаток этих подходов 
заключается в том, что эти методы требуют использовать сложное дополнительное оборудование в виде антенно-поворотного устройства, учитывать узкую направленность антенн и наличие специального программного обеспечения, а также зависимость от погодных условий или времени суток. Поэтому проведение такого рода экспериментальных исследований $\tau$ радиометрическим способом, который опирается на связь измеряемой $\mathrm{T}_{\mathrm{a}}$ с определяемой $\mathrm{T}_{\mathrm{b}}$ на основании выражения (10), следует считать более практичным и удобным.

Рассмотрение влияния перечисленных выше методов калибровки радиометров и нескольких способов определения рассеивающих свойств антенны на точность измерения $\mathrm{T}_{\mathrm{b}}$ атмосферы, а также расчет точности определения $\mathrm{T}_{\mathrm{b}}$ проведены для всех конкурирующих методов с помощью единого, хорошо известного подхода, который основан на использовании формул «переноса ошибок» $[1,16]$.

Для вычисления погрешностей определения $\mathrm{p}, \mathrm{T}_{\mathrm{a}}, \mathrm{T}_{\mathrm{b}}, \tau$ c помощью формул переноса ошибок были записаны соответствующие выражения. Например, для комбинации методов, когда калибровка проводится на основе охлаждаемой до азотных температур нагрузки, а параметры рассеяния АС определяются методом ИЛ, дисперсия погрешности определения

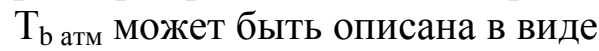

$$
\sigma_{\mathrm{T}_{\text {barм }}}{ }^{2}=\left(\frac{\partial \mathrm{T}_{\text {bатм }}}{\partial \alpha}\right)^{2} \sigma_{\alpha}^{2}+\left(\frac{\partial \mathrm{T}_{\text {bатм }}}{\partial \Delta \mathrm{n}}\right)^{2} \sigma_{\Delta \mathrm{n}}^{2}+\left(\frac{\partial \mathrm{T}_{\text {bатм }}}{\partial \beta}\right)^{2} \sigma_{\beta}^{2}+\left(\frac{\partial \mathrm{T}_{\text {bатм }}}{\partial \mathrm{T}_{\text {at }}}\right)^{2} \sigma_{\mathrm{T}_{\text {oat }}}^{2}+\left(\frac{\partial \mathrm{T}_{\text {bатм }}}{\partial \tau}\right)^{2} \sigma_{\tau}^{2},
$$

где $\sigma_{\mathrm{b}}$ атм - дисперсии погрешностей измерения или опенки соответствующих параметров.

Вычислив производные и перейдя к относительным среднеквадратическим погрешностям, можно получить выражение для расчета погрешности определения $\mathrm{T}_{\mathrm{b}}$ :

$$
\delta \mathrm{T}_{\mathrm{b} \text { атм }}{ }^{2}=\sigma \mathrm{T}_{\mathrm{b} \text { атм }}{ }^{2} \backslash\left(\mathrm{T}_{\mathrm{b} \text { атм }}\right)^{2} .
$$

Для измерения параметров рассеяния рупорной антенны радиометра была разработана специальная методика, основанная на использовании широко известного устройства - искусственной луны (ИЛ), в сочетании с применением специальной полой пенопластовой бленды с радиопоглощающим материалом, помещаемым в жидкий азот (рис. 15). Эта методика, позволяющая измерить радиометрическим методом к.п.д. $\eta$ и рассеяния антенны в неизотропную $\beta$ ni, изотропную $\beta$ і части ДН, которые расположены вне главного луча ДН антенны $\beta$, детально описана в [17].

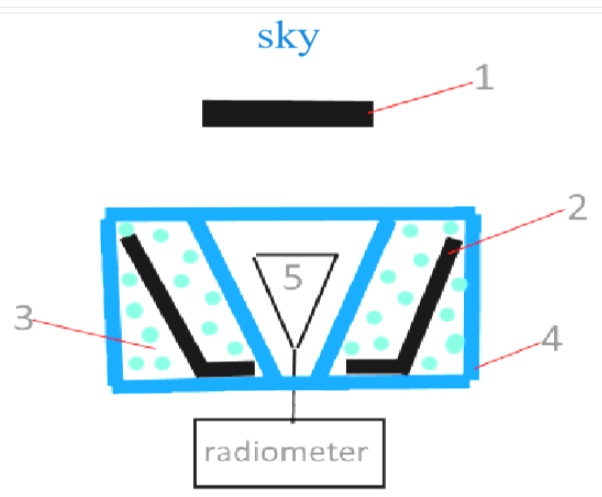

Рис. 15. Схема измерения параметров рассеяния рупорной антенны: 1 - ИЛ,

2 - бленда из аналога АЧТ, 3 - жидкий азот, 4 - пенопластовая емкость, 5 - исследуемый рупор

Исследование с помощью данного метода параметров направленности наиболее распространенных типов рупорных антенн [18] показало, что наименьшая величина параметра рассеяния была отмечена у рупоров с изломами угла раскрыва. Это тип рупора, ДН которого формируется коррекцией распределения амплитуды и фазы поля в раскрыве рупора за счет изменения угла раскрыва рупора. С помощью такого рупора и с использованием метода двух нагрузок проведены прецизионные измерения радиояркостной температуры зенитной области атмосферы. После чего с привлечением известного метода радиоизлучения леса [19] были измерены значения $\beta$ для предварительно отьюстированных двух экземпляров узконаправ- 
ленных антенн Кассегрена. Полученные в диапазоне 90 ГГц значения $\beta$ этих антенн с 2м апертурой составили 0,24 и 0,26 , в то время как значения параметра $\beta$ различных типов исследованных рупорных антенн [18] располагались в пределах 0,03-0,06.

Отметим, что, с технологической и бюджетной точек зрения, рупор с изменяемым углом раскрыва перспективный для его использования вплоть до частот 700 ГГц $[19,20]$. Однако для ММ, субММ ДВ (вплоть до ТГц частот) наиболее перспективно использование малоапертурных антенн, выполненных на основе открытого конца пенно-диэлектрической линии передач [21 - 23]. По совокупности своих диаграммо-образующих свойств (УБЛ, уровню кроссполяризационного излучения и уровню рассеяния вне основного лепестка ДН), а также по своей бюджетности и технологичности этот тип малоапертурных антенн [24] превосходит исследованные нами в диапазоне 100 ГГц аналоги [17].

Наряду с описанным способом, использующим охлаждаемую до азотных температур пенопластовую бленду с радиопоглощающим материалом, для оценок влияния на точность измерения $\mathrm{T}_{\mathrm{b}}$ атмосферы величины $\mathrm{p}$ были также рассмотрены методы:

а) «искусственной луны» [18];

б) «радиоизлучения леса» [18];

в) радиоизлучения центральной части лунного диска в период новолуния (применимый для остронаправленных зеркальных антенн).

В табл. 4 приведена оценка точности определения $T_{b}$ неба для различных методов калибровки радиометра и методов измерения параметра рассеяния антенной системы. Анализ продемонстрировал, что максимальную точность определения $\mathrm{T}_{\mathrm{b}}$ атмосферы (около $3 \%$ ) можно получить, калибруя радиометр с помощью охлаждаемой жидким азотом согласованной нагрузки облучателя, а также измеряя рассеяние антенны путем ее расположения в радиопоглощающей бленде и используя метод «искусственной луны», с помощью выражения

$$
(1-\beta) \mu=\mathrm{T}_{\mathrm{aam}}-\mathrm{T}_{\mathrm{az}} / \mathrm{T}_{\mathrm{oam}}-\mathrm{T}_{\mathrm{bz}}
$$

где $\mathrm{T}_{\text {aаm }}$ - антенная температура ИЛ перекрывающей основной лепесток ДН антенны; $\mathrm{T}_{\mathrm{az}}$ - антенная температура зенита; $\mathrm{T}_{\text {оат }}$ - термодинамическая температура радиопоглощающих материалов бленды и ИЛ; $\mathrm{T}_{\mathrm{bz}}$ - значение яркостной температуры зенита.

Таблица 4

\begin{tabular}{|l|c|c|}
\hline \multirow{2}{*}{ Методы измерения параметра $\beta$} & \multicolumn{2}{|c|}{ Методы калибровки } \\
\cline { 2 - 3 } & $\begin{array}{c}\text { Метод двух нагрузок } \\
(300 \mathrm{~K}, 78 \mathrm{~K})\end{array}$ & \begin{tabular}{c} 
Метод расчета радиояркости неба \\
\hline излучения Луны
\end{tabular} \\
\hline «искусственной луны» & $3 \%$ & $6 \%$ \\
\hline «излучения леса» & & $9 \%$ \\
\hline
\end{tabular}

Остальные комбинации рассмотренных методов обеспечивали более низкие значения точности. Для других рассмотренных методов калибровки радиометра это обстоятельство связано с неточностью оценок радиояркости источников космического излучения (для Луны в диапазоне 100 ГГц, - около 10 \% и наличие фактора влияния лунной фазы), а также это связано с ограниченной точностью определения радиояркости атмосферы по метеоданным (5 - $10 \%$ для случаев безоблачной атмосферы). Кроме того, точность методов калибровки и методов измерения параметров рассеяния антенн, которые используют пространственную (угломестную) переориентацию антенны, понижается за счет изменения интенсивности фона, принимаемого задними и боковыми лепестками ДН антенны.

Когда не требуется максимально возможная точность определения интенсивности радиотеплового излучения атмосферы или источника космического излучения целесообразно использовать более простые и удобные в реализации методы измерений. Например, проводить калибровку приемников по расчетному (на основании метеоданных) излучению зенит-

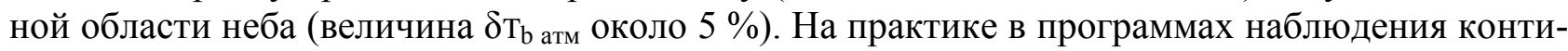
нуального излучения атмосферы использовались различные сочетания этих методов. 
Для наиболее точной из рассмотренных комбинаций, заключающейся в использовании метода ИЛ, а также жидкого азота для охлаждения аналогов АЧТ для калибровки радиометра и определения значения р его антенны (для параметров конкретной радиометрической аппаратуры диапазона 94 ГГц, описанной в п. 2.2) погрешности измерения составили для $\beta-9 \%, \alpha-1 \%, \mathrm{~T}_{\mathrm{b}}-3 \%$.

\section{Заключение}

Представлена трехчастотная $(12,40$ и 94 ГГц) радиометрическая система для исследования параметров полного вертикального ослабления атмосферы, ее влагозапаса и водозапаса.

Отработан (на основе серийного телевизионного конвертора) технически эффективный и экономичный подход для создания высокочувствительных неохлаждаемых радиометров диапазонов, расположенных в диапазоне 10 и 20 ГГц, которые могут найти применение в задачах метеорологии, вспомогательных коммуникационных задачах, а также в технологических и охранных приложениях.

Описаны методики радиометрических измерений радиояркости атмосферы и ослабления в ней радиоволн с качественным анализом источников ошибок и количественной оценкой точности различных комбинаций калибровочных методов радиометра и методов измерения коэффициента рассеяния антенны.

Показано, что эффективное использование диапазона 60 ГГц при развертывании сетей $5 \mathrm{G}$ на основе малых сот требует учитывать микроклиматические факторы.

Разработанное аппаратурно-методическое обеспечение для исследования радиофизических параметров атмосферы представляет интерес для задач метеорологии, климатологии и искусственного воздействия на облака, а также для определения параметров надежности линий спутниковой связи в рамках перспективного $5 \mathrm{G}$ формата.

\section{Список литературы:}

1. Радиотеплолокация в метеорологии / В.Д. Степаненко, Г.Г. Щукин, Л.П. Бобылев, С.Ю. Матросов. Ленинград : Гидрометеоиздат, 1987. $283 \mathrm{c}$.

2. Rec. ITU-R P.838-3, Specific attenuation model for rain for use in prediction methods.

3. Rec. ITU-R P.838 Definition of availability for radiocommunication circuits in the mobile-satellite service

4. Seybold J. Introduction to RF propagation. John Wiley \& Sons Inc., Hoboken, New Jersey, 2005. 2007.

5. Freeman R. L. Radio System Design for Telecommunications. John Wiley \& Sons Inc., Hoboken, New Jersey,

6. Boncho G. Bonev, Kliment N. Angelov and Emil S. Altimirski Study on Radio Link Availability in Millimeter Wave Range // World Academy of Science, Engineering and Technology. 2011. Vol.5. P.626-629.

7. Руженцев Н.В. Вертикальное атмосферное ослабление радиоволн миллиметрового диапазона. Изд-во LAMBERT Academic Publishing (Германия), 2015. 128 стр.

8. Liebe H.J. MPM - an atmospheric millimeter waves propagation model // International Journal of Infrared and Millimeter Waves. 1989. Vol.10, no 6. P. 631-650.

9. Martin Grabner, Vaclav Kvicera, Ondrej Fiser. Rain attenuation measurements and prediction on parallel 860$\mathrm{nm}$ free space optical and 58-GHz millimeter-wave path // Opt. Eng. 2012.Vol. 51, no. 3.

10. Ruzhentsev N.V. Compact $3 \mathrm{~mm}$ wave band transceivers for technologucal radars and radiotelephone communication systems // Turkish Journal of Physics. 1996. Vol.20, N4. P.356-358.

11. Odokienko O.V., Pavlikov V.V, Ruzhentsev N.V., Volosyuk V.K., Zhyla S.S. Two Frequency Radiometric Complex for Atmosphere Research // Proceedings of the 14-th International Conference Modern problems of radio engineering, telecommunications and computer science (TCSET'2018), Lviv-Slavsko, Ukraine, February 23 - 26, 2018, p. $28-31$.

12. Pavlikov V. V., Ruzhentsev N. V., Sobkolov A. D., Salnikov D. S. Tsopa, A. I. Ground-based radiometric complex of millimeter wave band for meteorology and telecommunications applications // Telecommunication and Radio Engineering. Begell House, 2017. Vol. 76, issue 16. P. 1477-14.

13. Tsopa A.I., Ivanov V.K., Leonidov V.I., Maleshenko Yu.I., Pavlikov V.V., Ruzhentsev N.V. and Zarudniy A.A. The research program of millimetric radio waves attenuation characteristics on perspective communication lines of Ukraine. 2016 13th International Conference on Modern Problems of Radio Engineering, Telecommunications and Computer Science (TCSET), Lviv, 2016. P. 638-642.

14. Strelnitskiy A.A., Strelnitskiy A.E., Tsopa O.I. and Shokalo V.M. Prediction model of energy security for the systems of subscriber radio access with branched street and corridor communications channels // Radioelectronics and Communications Systems, Allerton Press, Inc. 2011. Vol. 54, no. 2. P. 61-67.

15. Ruzhentsev N.V. and Churilov V.P. Terrain radiation - measurement investigation at 3-mm wave band // International Journal of Infrared and Millimeter Waves. 1996. V.17, N 2. P. 433-449. 
16. Худсон Д. Статистика для физиков. Москва : Мир, 1967. 242 с.

17. Ruzhentsev N. V. Complex Research of Parameters of Radiation of Axisymmetrical Antennas with Small Aperture at Millimeter Waves Band // Proc. of Int. Kharkov Symposium - Physics and Engineering of Microwaves, Millimeter and Submillimeter Waves (MSMW), June 21-24, Kharkov, Ukraine, 2016. 4 p.

18. Шифрин Я.С. Антенны. Харьков : ВИРТА им. Л.А. Говорова, 1976. 408 с.

19. Leech J., Tan B. K., Yassin G., Kittara P., Wangsuya S., Treuttel J., Henry M., Oldfield M. L., Huggard P. G. Multiple flare-angle horn feeds for sub-mm astronomy and cosmic microwave background experiments // Astron. Astrophys., vol. 532, p. A61, Aug. 2011.

20. Xing-Hui Yin, Sc Shi A simple design method of multimode horns // IEEE Transactions on Antennas and Propagation 53(1):455 - 459, february 2005.

21. Knyaz'kov L.B., Ruzhentsev N.V.Quasioptical polarization multipath diplexer // International Journal of Infrared and Millimeter Waves. 2006. V. 27, No.2. P. 211-217.

22. Knyaz'kov L. B. and Ruzhentsev N. V. Quasi-Optical Diplexer and Filter Based on a Polarization Ring Interferometer // ISSN 1063-7850 Technical Physics Letters. 2007. Vol. 33, No. 9. P. 761-763.

23. Knyaz'kov L. B. and N. V. Ruzhentsev N. V. Foamy-Dielectric Lens Transmission Lines for Millimeter and Submillimeter Wavelength Range // ISSN 1063-7850 Technical Physics Letters. 2008. Vol. 34, No.10. P. 888-890.

24. Knyaz'kov L. B. and Ruzhentsev N. V. Characteristics of the radiation from the end of the Transmission Line on the basis of spherical lenses made of foam dielectric // Proc. of Int. Kharkov Symposium - Physics and Engineering of Microwaves, Millimeter and Submillimeter Waves (MSMW), 2013.

Харьковский национальный 